\title{
Study of Myopia Correction Using Nanoporous Polymer Gas Permeable Contact Lenses
}

\author{
SILVIA SALAVASTRU ${ }^{1}$, IRINA ANDREEA NIAGU ${ }^{*}$, LUANA ANDREI MACOVEI', \\ LUCRETIA ANGHEL ${ }^{2}$, NICOLAE SARBU ${ }^{2}$, ALISA BEJAN ${ }^{1}$, \\ CLAUDIA FLORIDA COSTEA ${ }^{1 *}$, VICTORITA STEFANESCU ${ }^{2}$, \\ MADALINA NICOLETA MATEI ${ }^{2}$, CAMELIA MARGARETA BOGDANICI ${ }^{1}$ \\ 1 "Grigore T. Popa" University of Medicine and Pharmacy, Iasi, Faculty of Medicine, 16 Universitatii Str., 700115, Iasi, \\ Romania \\ 2 "Dunarea de Jos" University of Galati, Galati, Faculty of Medicine and Pharmacy, 47 Domneasca Str., 800008, Galati, \\ Romania
}

\begin{abstract}
Ophthalmic treatments using contact lenses are now used by more and more specialists around the world. Improving contact lens $(C L)$ materials is a condition that is constantly evolving. Contact lens materials are usually composed of polymer hydrogel or silicone hydrogel. The materials used for night contact lenses are gas permeable and they gradual flatten the center of the cornea which decreases the progression of myopia or myopic astigmatism. The aim of this study is to identify, in correlation with the chemical interactions between structural components of contact lenses and their biocompatibility with the surface layer and microtopography of the cornea or sclera, different incidents that occur in patients who have used rigid gas permeable contact lenses. The study was performed on a group of 10 patients who had a follow-up period between 4 and 6 years, who presented regularly for eye examinations. The following clinical parameters were analyzed: ocular refraction before and after wearing night contact lenses, types of contact lens materials, appearance of corneal topography, biomicroscopic examination of the anterior segment of the eye, keratometry, ocular comfort, as well as other incidents regarding this type of lenses. Difficulties caused by wearing contact lenses at night arose due to poor hygiene and maintenance in two cases or due to interruption of lens wear in one case.
\end{abstract}

Keywords: fluorosilicone acrylate, siloxanylstyrene fluoromethacrylate, crosslinked 3D structures, gaspermeable rigid contact lenses, wetting capacity, biocompatibility, myopia

\section{Introduction}

A contact lens (CL) is a device placed on the surface of the cornea or sclera to correct ametropia or it may be used for other ocular therapeutic purposes (eg, corneal healing).

The materials used to make contact lenses are usually composed of polymer hydrogel or silicone hydrogel. CLs were originally created from glass until the introduction of polymethyl methacrylate (PMMA) in the 1930s, which proved to have many benefits for maintaining proper corneal oxygenation. In 1936, a hybrid contact lens was designed, consisting of a central glass part corresponding to the cornea and a peripheral scleral PMMA portion. After this, the scleral lenses were created from PMMA for both central (corneal) and peripheral (scleral) portion [1].

After PMMA was found to cause corneal hypoxia, new materials were created. In 1937 the first gas permeable (GP) contact lens with cellulose acetate was developed. Silicone elastomers, created between 1960 and 1970, showed better oxygen permeability, but had a significant risk of deposit formation and were also very expensive. In 1974 cellulose acetate butyrate was introduced, it was softer than PMMA, but had low oxygen permeability. Further improvements included the addition of other components, such as styrene and fluorine, to improve biocompatibility, leading to the development of fluorosilicone acrylate material in 1987. GP contact lenses are usually created from fluorosilicone acrylates, have been and are still used for the treatment of keratoconus, for the correction of high astigmatism and in ortho keratology $[1,2]$. 
The concept of orthokeratology (orthoK) appeared in the early 1950s and demonstrated that in RGP (rigid gas permeable) contact lens wearers vision improved. Overnight use was approved by the FDA (Foods and Drugs Administration) in 2002 for Paragon CRT (corneal refraction therapy) lenses [2]. OrthoK reduces progressive myopia and / or myopic astigmatism, being recommended especially in children and adolescents and has an even greater benefit if initiated at an early age (6-8 years) [3]. Moreover, orthoK reduces the elongation of the antero-posterior axis in myopia [4].

Orthokeratology, although an effective procedure for correcting myopia, is still a fairly expensive technique, as it requires advanced technologies and special equipment that can be daunting for some families, who will certainly look for other ways of treatment [5]. Complication prevention requires the right selection of patients, the correct choice of lenses and the rapid achievement of stable positioning of the lenses, as well as proper care of them [6].

For using night contact lenses, it is mandatory to realise cornean topography before and during the treatment to monitorise the efficacy of aplanation [7].

Orthokeratology lens represent a gas permeable contact lens, whose curvature of the center is flatter than the periphery. OrthoK lenses improve temporary uncorrected visual acuity during the day, after using them overnight and removal upon wake up [8].

The materials used in orthoK lenses are presented in Table 1 [9].

Table 1. Materials used in orthoK lenses [9]

\begin{tabular}{|c|c|c|c|c|}
\hline Laboratories & Lenses & Materials & Dk (ISO) & Colour \\
\hline $\mathrm{LAO}$ & TopK & Boston XO2 & 141 & $\begin{array}{l}\text { RE :violet } \\
\text { LE: blue }\end{array}$ \\
\hline LCS & CRT & HDS 100 & 100 & dissociated colors \\
\hline MENICON & Z Night & $\mathrm{Z}$ & 163 & $\begin{array}{l}\text { RE : pink } \\
\text { LE : blue }\end{array}$ \\
\hline OPHTHALMIC & OverNight & Optimum extra & 100 & $\begin{array}{l}\text { RE : violet } \\
\text { LE : blue }\end{array}$ \\
\hline PRECILENS & $\begin{array}{c}\text { DRL } \\
\text { DreamLite } \\
\text { Sleep\&See } \\
\text { by Precilens }\end{array}$ & $\begin{array}{c}\text { Optimum 100/125 } \\
\text { Boston XO/XO2 } \\
\text { Boston XO } \\
\text { Boston XO/XO2 }\end{array}$ & $\begin{array}{c}100 / 25 \\
100 / 141 \\
100 \\
100 / 141\end{array}$ & $\begin{array}{l}\text { RE : violet } \\
\text { LE : blue } \\
\text { RE: blue } \\
\text { LE : violet }\end{array}$ \\
\hline
\end{tabular}

All materials used for night contact lenses (Boston XO or X02, HDS 100, Z, Optimum and Optimum extra) are composed by fluorosilicone acrylate [9].

Orthokeratology uses corneal topography to determine the shape of the cornea before treatment. The corneal topographer makes the "map" of the corneal convexity using thousands of points. Corneal topography helps to create customized lenses for each eye and patient, and also helps to monitorize the corneal flattening caused by wearing the contact lens.

Starting from the current state of studied materials used in the manufacture of GP contact lenses, the chemical interactions between structural components and their biocompatibility with the surface layer and microtopography of the cornea or sclera, various incidents that occurred during treatment were monitored. Moreover, a series of clinical parameters were analyzed: ocular refraction before and after wearing contact lenses at night, types of materials in the composition of contact lenses, appearance of corneal topography, biomicroscopic examination of the anterior segment of the eye, keratometry, eye comfort, as well as the incidents caused by these lenses. The study was performed on a group of 10 patients for a period of 4 to 6 years. 


\section{Materials and methods}

\subsection{Materials}

The study used correctly chosen contact lenses based on structural-functional characteristics with proper biocompatibility for any age group of patients. These were Menicon type, made of siloxanylstyrene fluoromethacrylate (Tisifilcon A), with good permeability to aqueous systems and Precilens type, theDouble Reservoir Lens (DRL), made of Boston XO2 material (hexafocon B).

As these materials are considered non-polar, but retain a good wettability and, above all, provide Dk values exceeding 100 bars. Therefore, the former has good hardness, while maintaining good wettability and a high Dk, while the second type has a softer surface, being more sensitive to scratches. The two types of lenses have different surface morphostructural characteristics, but each has advantages that allow them to be taken into account depending on the ophthalmic clinical parameters.

The slightly different characteristics of the two types of lenses lie in the formulae used in the manufacturing technology of the three components: fluoromethacrylate, methyl methacrylate and TRIS (tris (trimethyl-siloxy) -methacryloxy-propylsilane). The formulae adjustment allows the optimization of oxygen permeability, hardness or mechanical behavior and wettability. The last feature was improved by the inclusion of a hydroxyl group in the siloxy monomer [10]. Thus, in order to improve the wetting of the surface in the second type of CL, an itaconated ester (for example dimethyl itaconate) was incorporated into this monomer. Fluoroalkyl methacrylate together with siloxy methacrylate were used in both rigid lenses with increased oxygen permeability to improve the Dk characteristic. To enhance the permeability, in the formulae of the composition of methyl methacrylate homopolymers [11], it was replaced with one of the two fluorinated monomers: trifluoroethyl methacrylate (Menicon) and hexafluoroisopropyl methacrylate (Precilens). This process of adjusting the formulae of TRIS, as a means of obtaining 3D crosslinked rigid nanoporous materials for contact lenses with Dk values higher than PMMA, was one of refinement and improvement that led to the introduction by Menicon of new siloxanylstyrene monomers in the base polymer chain. Menicon material contains tris (trimethylsiloxy) silyl styrene as the key monomer for fluoromethacrylate. This chemical structure has excellent mechanical properties, allowing the CL to be significantly thinner than the typical rigid ones [12]. It was the first rigid lens material classified as "hyperoxygenic transmissibility" with a Dk of 175 and is the only FDA-approved rigid CL.

A high oxygen permeability was correlated with maintaining acceptable dimensional stability and ocular compatibility (characterized by moisture and resistance to deposition). Essential structural developments were focused on the incorporation of hydrophilic comonomers or on the subsequent modification of the formed lens surface and the use of crosslinking agents for soft lenses, with significantly improved watering characteristics.

Silicone hydrogel polymers fromsoft CL have been very successful, as they can be made with a water content between 0 and $60 \%$. At a low water content $(<10 \%)$, the polymers are rigid. In contrast, for rigid hydrogel silicone materials, the water content and expansion are limited by decreasing the ratio of hydrophilic monomers and increasing the density of the reticles. Thus, the polymer cannot absorb water inside the matrix, but the surface can be hydrated like a hydrogel lens. A second type of such polymer has been formulated, based on a hydrolyzable reactive monomer, which is included in the chain, often used in the manufacture of Precilens CL. This means that when the lens is placed in water, the reactive monomer hydrolyzes, producing a hydrophilic surface like a hydrogel lens. The reaction cannot continue inside because the expansion is constrained by crosslinking. This type also has thefeature of regenerating superficial hydrophilicity if the lens surface is damaged.

It is known that the oxygen permeability for Precilens type improves with increasing water content, and the dehydration rate tends to increase with water content. Instead, the higher hardness of the Menicon type offers better resistance to deposits. 


\subsection{Human subjects and study methods}

This paper represents a prospective study performed on a group of 10 patients (20 eyes) for whom orthokeratology was recommended, performed within the Ophthalmological Private Practice "Stereopsis", Iasi, with a six years follow-up interval (from 2014 to 2020).

The clinical parameters which were analyzed are the following: sex, ocular re-fraction at the first consult, ocular refraction after orthoK, visual acuity with the best correction, corneal topography, the type of contact lens (the type of material used), anterior segment of the eye examination, ocular and general comfort and different incidents or events.

The inclusion criteria are the following: patients over 7 years old, low or moder-ate myopia <-8D, myopic astigmatism with cylinder value $<2.50 \mathrm{D}$, absence of personal history of systemic or ocular allergies, acute inflammation of the anteri-or segment of the eye, dry eye syndrome, systemic underlying disease.

For the corneal topography we used Zeiss Atlas 9000 corneal topographer and Piccollo keratron. Both of them offer the possibility of objective interpretation of ocular surface modifications.

Axial (sagittal) topography helps us to make a quantitative assessment, in parallel with the subjective glasses correction (which is often confirmed). The tangential topography highlights the presence of a red ring that represents the decompression zone, and the green to blue zone is the compression zone. The existence of the red ring shows that the "night lens" had the desired efficiency $[13,14]$. Another used corneal topographer is Zeiss Atlas 9000 which can analyse different parameters such as axial and mean curvature, elevation or irregularity of the cornea, the pupil diameter, the steepest or the flatest meridian and the astigmatism.

The night lenses were changed at one year, according to the protocol, with reevaluation of all ocular parameters (visual acuity, refraction, topography).

The study was conducted in accordance with the principles of Declaration of Helsinki and the Local Ethics Committee for Scientific Research of the Grigore T. Popa University of Medicine and Pharmacy approved this study, after the informed consent has been read and signed by the patients or their parents. All patients went to the Stereopsis Clinic for optical correction with a "night lens" and were examined and followed up by a single specialist.

\section{Results and discussions}

The age of the patients included in the study is between 9 and 42 years with an average age of 13.9 $\pm 7.5,70 \%$ of them being females. Our results showed that $55 \%$ of patients had low myopia, $35 \%$ medium myopia and $10 \%$ myopic astigmatism.

The corneal topography was performed with difficulty in one case, because the patient had photophobia and excessive blinking and another patient needed to perform repeated corneal topography after wearing the contact lens, because the flattening was realised in the periphery of the cornea, due to the fact that the head position during the night was predominantly on that side (flattening of the cornea was not central). Difficulties caused by wearing night lenses occurred due to poor hygiene and maintenance in 2 cases and due to discontinuation of lens use (in one case).

Contact lenses were changed once a year 5 times in $30 \%$ of cases, 4 times in $50 \%$ of cases and 3 times in $20 \%$ of patients.

Figures 1 and 2 were realised with Piccolo Keratron for the analysis of corneal topography in axial and tangential incidence. Axial (sagittal) topography performs a quantitative evaluation, in parallel with the correction of the values given by autorefractometry as well as by the subjective correction for the prescription of glasses. The tangential topography highlights the presence of a red ring that represents the decompression zone, and the green to blue zone represents the compression zone. The existence of the red ring shows that the "night lens" had or did not have the desired efficiency $[13,14]$. 


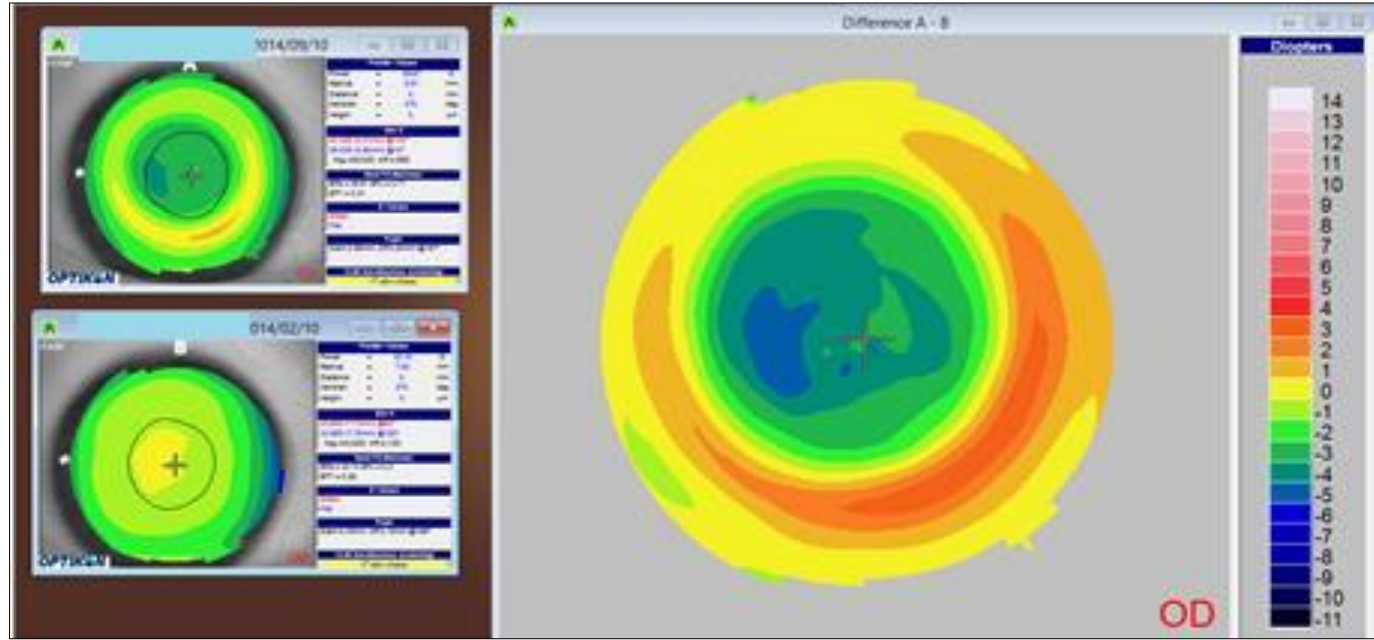

Figure 1. RE Axial topography - Piccolo keratron (personal case report)

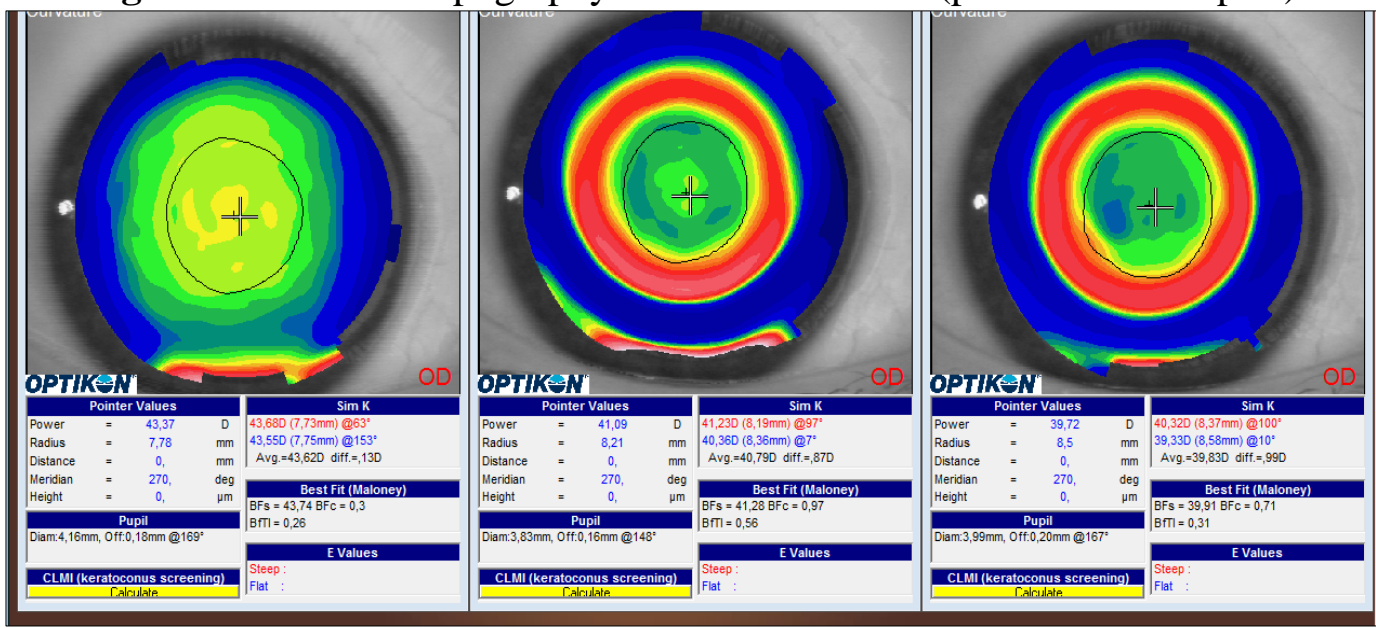

Figure 2. RE Tangential topography - Piccolo keratron (personal case report)

Compared to the Piccolo Keratron, the Zeiss Atlas 9000 corneal topographer can analyze other parameters, such as: axial and mean curvature, elevation or irregularity of the cornea, the pupil diameter, the steepest or the flatest meridian and the astigmatism (Figure 3).

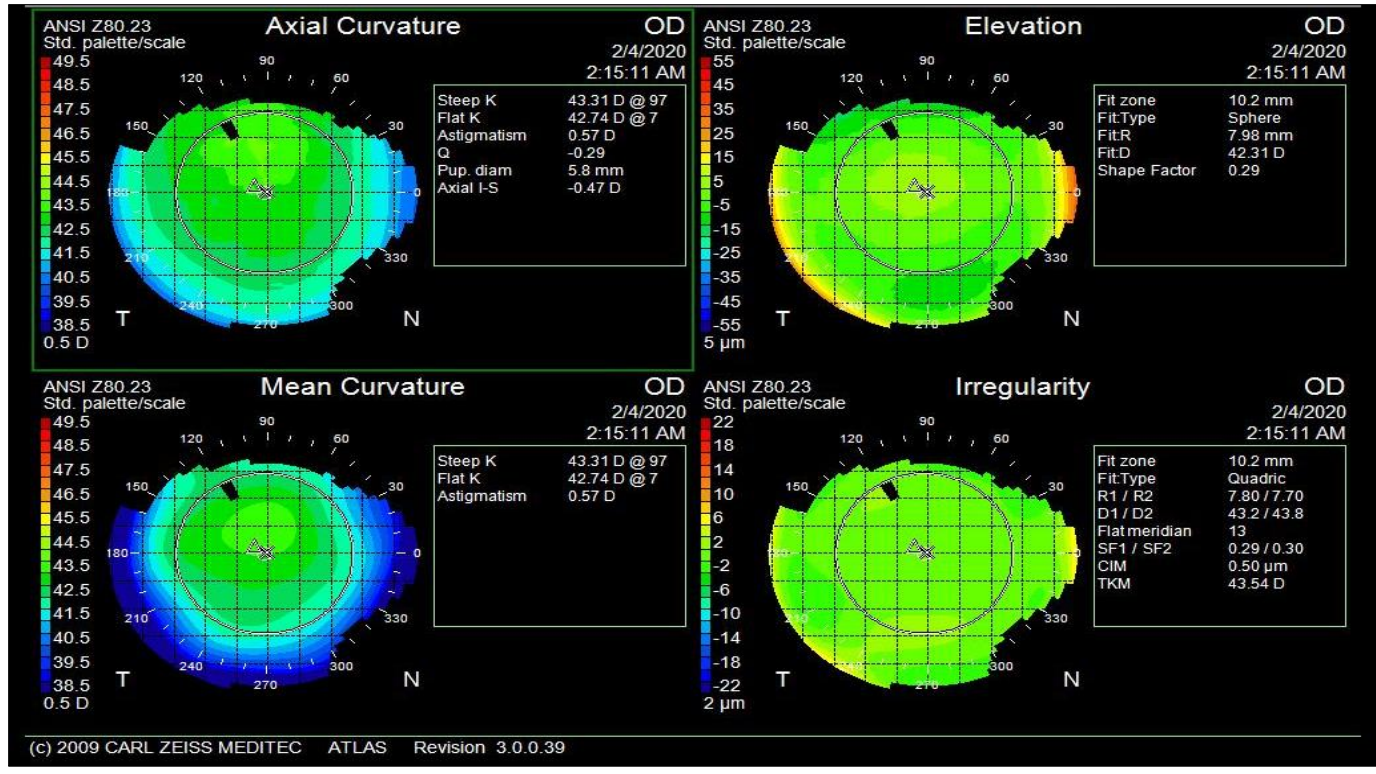

Figure 3. RE Corneal topography - Zeiss Atlas 9000 (personal case report) 
The change of refraction correlated with the appearance of the corneal topography determined at one day, one month and annually was determined for all patients. Evolution of corneal topography for right eye (Figure 4) and for left eye (Figure 5) shows the necessity of material change of night contact lenses after 5 years: siloxanilstiren fluoromethacrylate with fluorosilicone acrylate.

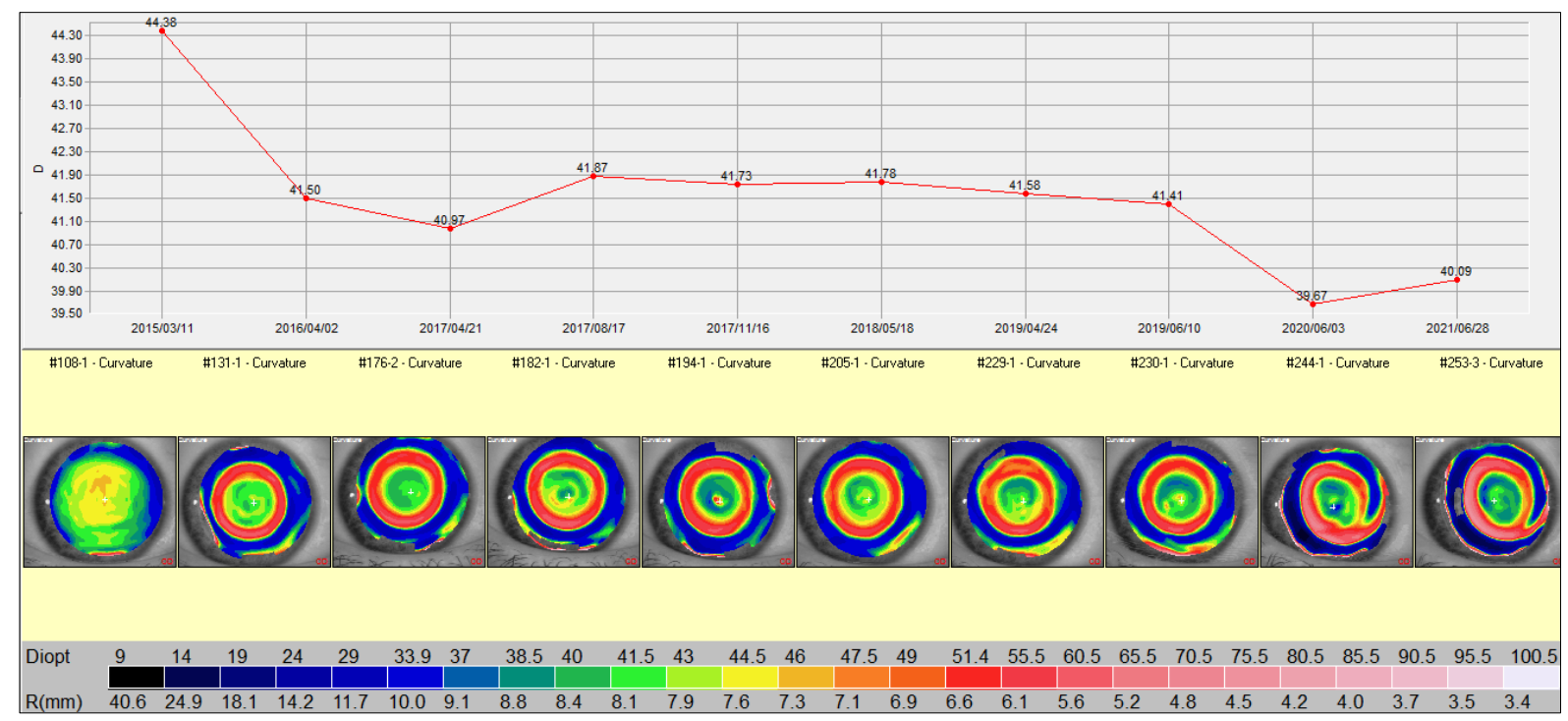

Figure 4. RE - Evolution of keratometry and corneal topography with Picollo keraton (personal case)

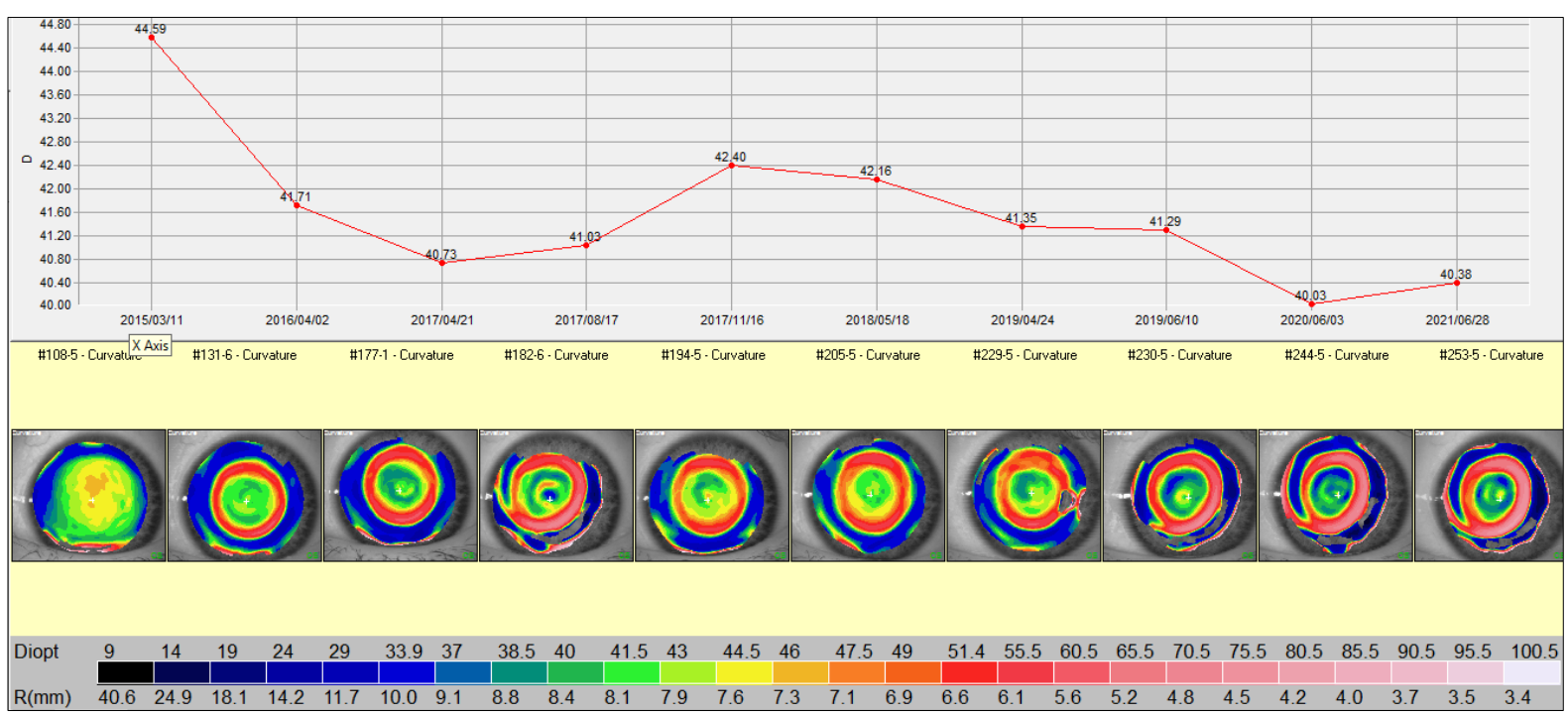

Figure 5. LE - Evolution of keratometry and corneal topography with Picollo keraton (personal case)

The prevalence of myopia in children is increasing worldwide and therefore new methods of correction are being searched, which should be accepted especially by the child. Excepting orthokeratology, atropine therapy has been shown to be effective in slowing the progression of myopia in children. Although the action mechanism of atropine is not fully understood, it has been shown to play a role in decreasing the diopter value and maintaining a normal visual acuity. Currently, it is recommended to combine the use of atropine and orthokeratology or to use only one method. Thus, a combined therapy of orthoK and atropine could have a great potential to reduce the progression of myopia [15]. 
In this study we did not use atropine therapy, only orthoK treatment. Orthokeratology is used today mainly for the correction of myopia up to $6.00 \mathrm{D}$, but it can also be used above these values. More importantly, orthoK is a major therapy for slowing the progression of myopia in children, and its effectiveness has been demonstrated in numerous studies [16]. The patients in our study fall within the recommendations for orthokeratology.

The two types lenses used for the patients in this study, Menicon and Precilens, the first, as stated, is made of a material called siloxanylstyrene fluoromethacrylate (Tisifilcon A) which increases the hardness and durability of the lens, while maintaining good permeability to water [17]. The second type of lens, Precilens, is a double reservoir lens (DRL) that is made of Boston XO2 material (hexafocon B) with an oxygen permeability of 141 barriers [18].

The biocompatibility of the substance has a major importance for the ocular safety. A significant feature of contact lens materials is their capacity to keep its physical characteristics after being exposed to various external conditions. The powerful water plasticizing effect from the silicone-hydrogel lens materials suggests a drop in intermolecular forces over the polymer chains, which increases the chain mobility and flexibility. In a study conducted in 2019, where two daily disposable (nesofilcon A and delefilcon A) and two monthly CL (comfilcon A and lotrafilcon B) were used, no significant modifications were observed in the physicochemical composition of the materials after dehydration and rehydration, suggesting a favorable stability of their components. [19].

The benefit of RGP lenses consists in their rigidity which produce reshaping of the cornea. Fluorosilicone-acrylates now make up the majority of RGP lense being fitted [20]. In a study conducted by Yang and co. showed that, by changing both the length of the fluorinated side chain and its final shape, the surface properties of the polymers that make up the composition of hard contact lenses are greatly improved. Thus, they have an optimal surface tension in the processes of surface wetting in aqueous environments, without affecting the resulting surface energy. The crosslinking structure of these polymers increases the mechanical robustness and offers a better surface stability and an optimal adhesion of the polymer to the substrate [21]. Experimental results show that such systems obtained in the form of copolymers with controlled molecular weights and narrow polydispersities, have high stability in aqueous dispersed media, also have very good thermal and wetting properties, compared to other copolymers used in the field of contact lenses [22].

In a study conducted by Tomic and co. on RGP contact lens materials, aquagrams showed that there exists a particular influence of the materials on saline. Infrared spectroscopy (aquaphotomics) method demonstrated typical influences of nanophotonic materials on saline solution [23].

In our study, three of the ten patients received lenses made of hexafocon B material. One of them, a 15-year-old woman with refraction RE: $-7 \mathrm{sph}$ and LE: $-8 \mathrm{sph}$, received this type of lens as the first treatment because it exceeds the value of $6 \mathrm{D}$. The patient had after a month's wear a normal visiual acuity and a decrease in diopters (but not up to 0 ), maintaining a residual value of $1 \mathrm{D}$.

A study conducted in 2015 showed that orthoK is useful in controlling or delaying the high progression of myopia [24]. From our case study, in one patient - with a constant refraction RE: -5.50 sph and LE: -5.75 sph- the Menicon lens was replaced with the Precilens lens. A 42-year-old patient who underwent refractive surgery on both eyes prior to wearing the night lens, but in whom myopia increased postoperatively, was recommended to wear the Precilens lens first. In 2020, in a study of adults with myopia, it was shown that orthoK is a safe and well-accepted option for myopic correction in adults [25].

In another study published in 2005, conducted in Hong Kong on 35 children using orthoK, involving a control group (also 35 children) who used monofocal glasses, showed that the average increase in axial length in the orthokeratology group was significantly lower than in the control group, showing a reduction rate of $46 \%$ induced by orthoK [26].

The efficiency of orthoK therapy was also successfully demonstrated by the Retardation of Myopia in Orthokeratology (ROMIO) study, in which 102 children with ages between 6 and 10 years old, were 
divided into orthoK and single-vision spectacle lenses group.The results showed a reduction rate of $43 \%$ after a two-year therapy with orthoK [27].

Another study conducted during a period of 12 years showed that orthoK was efficient in slowing myopia progression. In contrast with control group, orthoK group had a significantly lower refractive error change during the follow-up periods [28].

Similar to the mentioned studies, our results showed that orthoK therapy had an important role in slowing the myopic progression in children and also in adults.

In a study from 2016, none of the participants presented any important ocular complications after orthoK. One patient had some difficulties adjusting with the lens in the first couple months, most of the participants complied with all hy-giene measures and all the participants improved their attitude and became more confident and sociable with the help of the night contact lenses [13]. All these aspects are similar with our findings.

Studies have shown that using Menicon Z-Night contact lenses can be beneficial in correcting high corneal astigmatism $[29,30]$.

One of the most frequent complication of orthoK lenses is corneal staining, but this was not found in our study. Other complications are prominent fribrillary deposits or transient changes of corneal biomechanical properties [31], which also did not occur in our patients.

In a study conducted on 3800 eyes $8.8 \%$ had visual acuity problems due to discontinuous wear and $4 \%$ of them wore the contact lenses only for a short pe-riod of time [32], which are similar with problems found for our patients (who we excluded from this study, due to non-compliance with the minimum 6 hours time of the CL wear).

\section{Conclusions}

Based on the experimental data obtained, the following conclusions can be drawn:

- The diversity of materials used in orthoK contact lenses allow their change when modifications in refraction or corneal curvature are observed.

- Modern night lenses offer good ophthalmic treatment and bring benefits in both quality of vision and life for the patients with myopia or myopic astigmatism.

- OrthoK therapy has been shown to be effective in slowing the progression of myopia without the occurrence of severe complications.

\section{References}

1.PILLAY, R., HANSRAJ, R., RAMPERSAD, N., Historical Development, Ap-plications and Advances in Materials Used in Spectacle Lenses and Contact Lenses. Clin Optom (Auckl), 29(12), 2020, 157-167. doi: 10.2147/OPTO.S257081.

2.VANDERVEEN, D.K., KRAKER, R.T., PINELES, S.L., HUTCHINSON, A.K., WILSON, L.B., GALVIN, J.A., LAMBERT, S.R., Use of Orthokeratology for the Prevention of Myopic Progression in Children: A Report by the American Academy of Ophthalmology. Ophthalmology, 126(4), 2019, 623636. doi: 10.1016/j.ophtha.2018.11.026.

3.LI, X., FRIEDMAN, I.B., MEDOW, N.B., ZHANG, C., Update on Or-thokeratology in Managing Progressive Myopia in Children: Efficacy, Mecha-nisms, and Concerns. J Pediatr Ophthalmol Strabismus, 54(3), 2017, 142-148. doi: 10.3928/01913913-20170106-01.

4.LAU, J.K., VINCENT, S.J., CHEUNG, S.W., CHO, P., Higher-Order Aberra-tions and Axial Elongation in Myopic Children Treated with Orthokeratology. Invest Ophthalmol Vis Sci, 61(2), 2020, 22. doi: 10.1167/iovs.61.2.22.

5.NELSON, L.B., Orthokeratology Treatment in Pediatric Myopia. J Pediatr Ophthalmol Strabismus, 54(3), 2017, 141. doi: 10.3928/01913913-20170424-01.

6. LIU, Y.M., XIE, P., The Safety of Orthokeratology-A Systematic Review. Eye Contact Lenses, 42(1), 2016, 35-42. doi: 10.1097/ICL.0000000000000219. 
7. HAGE, S.E., LEACH, N.E., MILLER, W., PRAGER, T.C., MARSACK, J., PARKER, K., MINAVI, A., GAUME, A., Empirical Advanced Orthokeratology Through Corneal Topography: The University of Houston Clinical Study, Eye Contact Lenses, 33(5), 2007, 224-235.

8.CHEW, S., Toric Orthokeratology Effectiveness on Correcting Astigmatism: A Narrative Literature Review, JOJ Ophthalmology, 7, 2018.

9. BLOISE, L., Les avancees en contactologie, Edition Med-line, 2019, p. 137-146.

10.TANAKA, K., TAKAHASHI, K., KANADA, M., et al., Copolymer for Soft Contact Lens, Its Preparation and Soft Contact Lens Made Therefrom, US Patent, 1979,4139513.

11.GAYLORD, N.G., Oxygen Permeable Contact Lens Composition Methods and Article of Manufacture (To Polycon Lab Inc.), US Patent, 1974, 3808178.

12. SZCZOTKA-FLYNN, L., DIAZ, M., Risk of Corneal Inflammatory Events with Silicone Hydrogel and Low Dk Hydrogel Extended Contact Lens Wear: A Meta-Analysis, Optom Vis Sci, 84(4), 2007, 247-56.

13.BOGDANICI, C., BOGDANICI, S.T., TIUTIUCA, C., FERARU, C., New Materials Used for Vision - Orthokeratology, Mater. Plast. 53(3), 2016, 530-532.

14. KOTB, M., EISSA, S.A., Correlation Between Myopic Refractive Error, Corneal Power and Central Corneal Thickness in the Egyptian Population, Clin Ophthalmol, 15, 2021, 1557-1566.

doi:10.2147/OPTH.S304693.

15.TONE, S., NIAGU, I.A., BOGDANICI, S.T., BOGDANICI, C.M., Update in pediatric myopia treatment strategies, Rom J Ophthalmol, 64(3), 2020, 233-238.

16. CHO, P., TAN, Q., Myopia and orthokeratology for myopia control, Clin Exp Optom, 102(4), 2019, 364-377. doi: 10.1111/cxo.12839.

17.SANTODOMINGO-RUBIDO, J., VILLA-COLLAR， C., GILMARTIN, B., GUTIÉRREZORTEGA, R., Myopia Control with Orthokeratology Contact Lenses in Spain (MCOS): Study Design and General Baseline Characteristics, J Optom., 2(4), 2009, 215-22. doi: 10.3921/joptom.2009.215.

18.PAUNÉ, J., MORALES, H., ARMENGOL, J., QUEVEDO. L., FARIA-RIBEIRO, M., GONZÁLEZ-MÉIJOME, J.M., Myopia Control with a Novel Peripheral Gradient Soft Lens and Orthokeratology: A 2-Year Clinical Trial, Biomed Res Int., 2015, 2015, 507572.

doi: $10.1155 / 2015 / 507572$.

19.LIRA, M., LOURENÇO, C., SILVA, M., BOTELHO, G., Physicochemical stability of contact lenses materials for biomedical applications, J Optom, 13(2), 2020, 120-127.

doi:10.1016/j.optom.2019.10.002

20.MUSGRAVE, C., FANG, F., Contact Lens Materials: A Materials Science Perspective. Materials (Basel, Switzerland), 12(2), 2019, 261. https://doi.org/10.3390/ma12020261.

21.YANG, S., WANG, J.G., OGINO, K., VALIYAVEETTIL, S., OBER, C.K., Low-surface-energy fluoromethacrylate block copolymers with patternable elements, Chemistry of Materials, 12(1), 2000, 33-40. doi: $10.1021 / \mathrm{cm} 990241+$

22. GUO, T.Y., TANG, D., SONG, M., ZHANG, B., Copolymerizations of butyl methacrylates via RAFT miniemulsion polymerization, Journal of Polymer Science Part A-Polymer Chemistry, 45(22), 2007, 5067-5075. doi: 10.1002/pola.22249.

23.TOMIĆ, M., MUNĆAN, J., STAMENKOVIĆ, D., JOKANOVIĆ, M., MATIJA, L., Biocompatibility and cytotoxicity study of nanophotonic rigid gas permeable contact lens material, J. Phys. Conf. Ser., 2013, 429.

24.ZHOU, J., XIE, P., WANG, D., GUO, X., YANG, L., The long-term clinical effects of orthokeratology in high myopia children, Zhonghua Yan Ke Za Zhi, 51(7), 2015, 515-9.

25.REN, Q., YANG, B., LIU, L., CHO, P., Orthokeratology in adults and factors affecting success: Study design and preliminary results, Cont Lens Anterior Eye, 43(6), 2020, 595-601.

26.CHO, P., CHEUNG, S.W., EDWARDS, M., The longitudinal orthokeratology research in children (LORIC) in Hong Kong: a pilot study on refractive changes and myopiccontrol, Curr Eye Res, 30, 2005, 71-80. 
27.CHO, P., CHEUNG, S.W., Retardation of myopia in Ortho-keratology (ROMIO) study: a 2-year randomized clinical trial, Invest Ophthalmol Vis Sci, 53, 2012, 7077-7085.

28. LEE, Y.C., WANG, J.H., CHIU, C.J., Effect of Orthokeratology on myopia progression: twelveyear results of a retrospective cohort study, BMC Ophthalmol, 17(1), 2017, 243.

doi: 10.1186/s12886-017-0639-4.

29.CHAN, B., CHO, P., DE VECHT, A., Toric orthokeratology: a case report, Clin Exp Optom, 92(4), 2009, 387-91. doi: 10.1111/j.1444-0938.2009.00363.x.

30. PAUNÉ, J., CARDONA, G., QUEVEDO, L., Toric double tear reservoir con-tact lens in orthokeratology for astigmatism, Eye Contact Lenses, 38(4), 2012, 245-51.

doi: 10.1097/ICL.0b013e318258789e.

31. LIU, Y.M., XIE, P., The Safety of Orthokeratology - A Systematic Review, Eye Contact Lenses, 42(1), 2016, 35-42. doi: 10.1097/ICL.0000000000000219.

32.JIWEN, Y., CAN, C., CHAO, Z., JIANG, L., YONGCHUAN, H., ZHEN, F., JIANHUA, L., ZIDAN, L., The clinical study of orthokeratology complications and the discontinue reasons, Invest Ophthalmol Vis Sci, 60(9), 2019, 6331.

Manuscript received: 30.09 .2021 